\title{
Urinalysis, but not blood biochemistry, detects the early renal-impairment in
}

\section{patients with COVID-19}

\author{
Haifeng Zhou ${ }^{\text {l\# }}$, Zili Zhang ${ }^{1 / \#}$, Heng Fan ${ }^{1 \#}$, Junyi $\mathrm{Li}^{1}$, Mingyue $\mathrm{Li}^{1}$, Yalan Dong ${ }^{1}$, Weina Guo ${ }^{1}$, Lan Lin $^{1}$, \\ Zhenyu Kang ${ }^{1}$, Ting $\mathrm{Yu}^{1}$, Chunxia Tian ${ }^{1}$, Yang Gui ${ }^{1}$, Renjie Qin ${ }^{1}$, Haijun Wang ${ }^{2}$, Shanshan Luo $^{3 *}$, \\ Desheng $\mathrm{Hu}^{1,3 *}$
}

${ }^{1}$ Department of Integrated Traditional Chinese and Western Medicine, Union Hospital, Tongji Medical College, Huazhong University of Science and Technology, Wuhan 430022, China (D Hu PhD, H Fan PhD, H Zhou MM, Z Zhang MM, J Li MM, Y Dong PhD, W Guo PhD, L Lin PhD, Z Kang PhD, T Yu PhD, C Tian MM, Y Gui MM, R Qin MM)

${ }^{2}$ Department of Neurosurgery, Union Hospital, Tongji Medical College, Huazhong University of Science and Technology, Wuhan 430022, China (H Wang PhD)

${ }^{3}$ Institute of Hematology, Union Hospital, Tongji Medical College, Huazhong University of Science and Technology, Wuhan 430022, China (D Hu PhD, S Luo PhD)

${ }^{\#}$ These authors contributed equally.

*Correspondence author:

Desheng $\mathrm{Hu}, \mathrm{PhD}$

Department of Integrated Traditional Chinese and Western Medicine, Union Hospital, Tongji Medical College, Huazhong University of Science and Technology, Wuhan 430022, China.

Tel: +86-27-8572-6395

Email: desheng.hu@ hust.edu.cn

Or Shanshan Luo, PhD 
Institute of Hematology, Union Hospital, Tongji Medical College, Huazhong University of Science and Technology, Wuhan 430022, China

Email: shsh689@126.com

\section{Abstract}

Background In December 2019, a novel coronavirus (SARS-CoV-2) caused infectious disease, termed COVID-19, outbroke in Wuhan, China. COVID-19 patients manifested as lung injury with complications in other organs, such as liver, heart, gastrointestinal tract, especially for severe cases. However, whether COVID-19 causes significant acute kidney injury (AKI) remained controversial.

Methods We retrospectively analyzed the clinical characteristics, urine and blood routine tests and other laboratory parameters of hospitalized COVID-19 patients in Wuhan Union Hospital.

Findings 178 patients, admitted to Wuhan Union hospital from February 02 to February 29, 2020, were included in this study. No patient $(0[0 \%])$ presented increased serum creatinine (Scr), and $5(2.8 \%)$ patients showed increased blood urea nitrogen (BUN), indicating few cases with "kidney dysfunction". However, for patients (83) with no history of kidney disease who received routine urine test upon hospitalization, $45(54.2 \%)$ patients displayed abnormality in urinalysis, such as proteinuria, hematuria and leukocyturia, while none of the patients was recorded to have acute kidney injury (AKI) throughout the study. Meanwhile, the patients with abnormal urinalysis usually had worse disease progression reflecting by laboratory parameters presentations, including markers of liver injury, inflammation, and coagulation.

Conclusion Many patients manifested by abnormal urinalysis on admission, including proteinuria or hematuria. Our results revealed that urinalysis is better in unveiling potential kidney impairment of 
COVID-19 patients than blood chemistry test and urinalysis could be used to reflect and predict the

disease severity. We therefore recommend pay more attention in urinalysis and kidney impairment in COVID-19 patients.

Keywords: COVID-19; kidney impairment; acute kidney injury; urinalysis; disease severity 


\section{Introduction}

A novel coronavirus, named SARS-CoV-2, caused pneumonia outbreak in December 2019 in Wuhan,

China, and quickly spread across the country. Until March 16, 2020, there have been 16,7511 confirmed cases of COVID-19 and 6606 deaths worldwide (announced by WHO). Despite most cases were from China from the beginning, local outbreak has occurred in other countries recently, such as Japan, South Korea, Iran and Italy. Epidemiological investigations demonstrated that elderly men were susceptible to the disease and more likely to develop into severe or critically ill stage(1-3). Furthermore, comorbidities, such as diabetes, hypertension, cardiovascular disease and cancers, are risk factors for COVID-19(1, 2). Though most of the COVID-19 patients usually initiated with flu-like symptoms, some of them underwent rapid deterioration and even die suddenly(4, 5). Emergency measures including quarantine, traffic control and suspension of public activities are implemented to limit the epidemic. However, there is no effective treatment against COVID-19 and the presence of asymptomatic carriers and the possibility of relapse impose additional challenges for COVID-19 prevention $(6,7)$.

Genomic sequence analysis indicated that SARS-CoV-2 is $96 \%$ identical to bat coronavirus and has $75 \%-80 \%$ similarity to severe acute respiratory syndrome coronavirus (SARS-CoV) (8-10). SARS-CoV-2 uses angiotensin converting enzyme II (ACE2) as cell entry receptor, thus, patients with COVID-19 could develop multiple organ or tissue injuries(11-14). It is reported that the patients with SARS-CoV-2 infection show elevated levels of alanine aminotransferase (ALT), aspartate aminotransferase (AST) and $\gamma \square$ glutamyl transferase (GGT), indicating liver and myocardial damage(1, 3, 15-17). Yet the existence of COVID-19 induced kidney impairment or dysfunction 
remain controversial. A recent study reported that $29 \%$ of the 52 patients with severe SARS-CoV-2 pneumonia were complicated with acute kidney injury (AKI)(4). Another study among 138 hospitalized patients with confirmed COVID-19 revealed that 5 patients (3.6\%) developed AKI and 3 of them ended up in the ICU. Yan et al. demonstrated that among the 59 COVID-19 patients with kidney impairment, 63\% exhibited proteinuria, 19\% displayed elevated level of plasma creatinine and $27 \%$ showed increased level of urea nitrogen(18). A study from Xu's group reported that 5.1\% (36/701) patients were identified as AKI(19). In addition, Huang et al revealed that AKI occurred in 7\% (3/41) patients(2). On the contrary, a study with 1099 confirmed COVID-19 cases indicated that only 6 patients $(0.5 \%)$ developed AKI(17). Another study conducted by Zhou et al revealed that only $4 \%$ (8/186) patients had increased level of Scr(1). Gong et al. reported that SARS-CoV-2 infection could neither cause obvious AKI nor aggravate CRF in the COVID-19 patients(20). Thus, as with other known extrapulmonary organ injury, the existence of AKI deserves to be disclosed in COVID-19 patients, which is pivotal in the disease diagnosis, treatment and prognosis.

The current study retrospectively analyzed the clinical data of 178 hospitalized COVID-19-confirmed patients in Wuhan Union Hospital and proposes to provide additional evidence on the incidence of kidney injury in COVID-19 patients and address a better indicator for the potential kidney impairment.

\section{Method}

\section{Study design}

178 patients with confirmed COVID-19 in Wuhan Union hospital, Tongji Medical College, Huazhong

University of Science and Technology, from February 02 to February 29, 2020, were enrolled in this 
retrospective study. All patients in this study were diagnosed according to the guidance (fifth edition)

published by Chinese National Health Commission. The patients with a history of kidney diseases were excluded from this study, and the flow chart was shown in Figure 1. And the clinical outcomes were monitored up to February 29, 2020. This study was approved by the Institutional Ethics Board of Wuhan Union Hospital of Tongji Medical College, Huazhong University of Science and Technology (No. Union Hospital-0093). Written informed consent was waived by the Ethics Commission of the designated hospital for the emerging infectious diseases.

\section{Data collection}

Demographic data, symptoms and vital signs, laboratory examinations were retrieved from electronic medical records for this study. The laboratory examinations contain blood routine test, blood biochemical tests (liver function, renal function, lipids and glucose), blood coagulation index, lymphocyte subsets and cytokines analysis, urine routine test, C-reactive protein (CRP), erythrocyte sedimentation (ESR), serum ferritin (SF), etc. Estimated glomerular filtration rate (eGFR) was calculated with Chronic Kidney Disease Epidemiology Collaboration (CKD-EPI) equation(21). Patients were daily assessed the presence of AKI or renal failure using strictly criteria based on the 2011 kidney disease: improved global outcomes (KDIGO) for AKI. All the laboratory measures were performed at the department of clinical laboratory in Union Hospital and the normal range of these measures were given by them. The threshold of Scr was 54-133 ( $\mu \mathrm{mol} / \mathrm{L})$ for male and 44-106 ( $\mu$ mol/L)

for female, BUN was 2.9-8.2 (mmol/L) , serum uric acid was 208-428 ( $\mu \mathrm{mol} / \mathrm{L})$ for male and 155-357 $(\mu \mathrm{mol} / \mathrm{L})$ for female, as well as Cystatin C was 0.63-1.25 (mg/L) for male and 0.54-1.15 (mg/L) for female. Proteinuria was defined as positive in urine protein (semiquantitative, $+-/+/++/+++$ ) and 
hematuria was recorded with elevated urine erythrocyte quantification (>17/ul) as well as positive in semiquantitative urinalysis $(+-/+/++/+++)$. All the parameters were analyzed using extreme values, except for urinalysis upon first examination on admission due to limited data.

\section{Statistical analysis}

The categorical variables were summarized as counts or percentages, and continuous variables were expressed as mean \pm SD or medians and interquartile ranges (IQR) as appropriate. Categorical variables were compared using the $\chi^{2}$ test; the Fisher exact test was used when the data were limited. Continuous variables were compared using independent group t test when the data were normally distributed; otherwise, the Mann-Whitney test was used. All statistical analyses were performed using SPSS 20.0 software, and $\mathrm{P}<0.05$ was regarded as a significant difference.

\section{Result}

\section{COVID-19 patients were seldom diagnosed with AKI}

A total of 178 patients with laboratory confirmed SARS-CoV-2 infection were enrolled in this study, among which 52 patients were from ICU and 126 patients were from ordinary isolation ward. As shown in Table 1, the median age of patients in ICU group was 56.5 years (IQR, 40.50-65.5), which was significantly higher than that of patients in non-ICU group (45, IQR [32.75-57.00]). The proportion of males in ICU group was higher than that of non-ICU group (61.5\% vs. $31.7 \%)$. More patients in ICU group rather than non-ICU group manifested high body temperature (T>38 $\square)(75 \%$ vs. 56.3\%). Additionally, some underlying diseases were more common in patients of ICU, such as hypertension ( $28.8 \%$ vs. $11.1 \%$ ), diabetes (17.3\% vs. $7.9 \%)$, cardiovascular insults (13.5\% vs. $2.4 \%$ ), 
digestive system $(7.7 \%$ vs. $4.8 \%)$ and respiratory system disease $(7.7 \%$ vs. $4.8 \%)$. These data were consistent with recent reports related to COVID-19 that elder men with comorbidities are more likely to be infected and develop into severe cases and even die due to weaker immune function.

Previous studies showed that many patients with SARS-CoV-2 infection usually had increased level of Scr, BUN and even AKI, especially those severe patients. Unexpectedly, these clinical manifestations, however, didn't appear in our data. Patients in both ICU group and non-ICU group displayed normal Scr and cystatin C level. Of all cases, although $23.6 \%$ patients showed decreased estimated glomerular filtration rate (eGFR) compared to normal level, only $2.8 \%$ patients were recorded with elevated level of BUN. Consistent with these results, none of patients develop acute kidney injury or acute renal failure during whole hospitalization regardless of entry into ICU. Taken together, our data supported that there was no significant kidney dysfunction among COVID-19 patients.

\section{Potential kidney impairment reflected by routine urine test}

Based on the fact that Scr and BUN may not be affected owing to strong compensatory adaption of kidney, urine examination results were collected to further evaluate the kidney impairment in detail. Not every COVID-19 patient received regular urine routine test. Only 87 of 178 enrolled patients underwent urine test on admission, among whom 4 patients were documented with history of kidney diseases: diabetic nephropathy, kidney stones, multiple renal cystic disease and kidney carcinoma. And

they were excluded in our cohort. The remaining 83 patients were selected for further analysis as illustrated in Figure 1, with a participation rate of $46.6 \%$. Of the 83 patients, no one reported related symptoms or was diagnosed as urinary system infection, urethral injury or other urinary system 
diseases. Unexpectedly, 54.2\% (45/83) patients showed abnormal urine test results, including proteinuria, hematuria, leukocyturia (Table 2). Specifically, 34.9\% cases had positive urine protein, with $15.7 \%$ for "+-", $16.9 \%$ for "+" and 2.4\% for "++" (Figure 2A). At the same time, 31.3\% patients presented with hematuria, including $2.4 \%$ for “+-", $16.9 \%$ for “+”, $8.4 \%$ for “++" and $3.6 \%$ for "+++"

(Figure 2A). In addition, the patients in ICU exhibited more severe urinalysis results compared with non-ICU patients, such as proteinuria (+, $31.6 \%$ vs. $12.5 \% ;++, 10.5 \%$ vs. 0$)$ and hematuria $(+, 26.3 \%$ vs. $18.8 \% ;++, 21.1 \%$ vs. $4.7 \% ;+++, 5.3 \%$ vs. $3.1 \%)(\mathrm{P}<0.05$ for all) (Figure 2B, C). These results indicated prevalence of kidney impairment among COVID-19 patients even without significant changes of filtration function.

\section{Kidney impairment were caused by direct virus invading}

Given that proteinuria and hematuria were two prominent signs of kidney impairment, 83 patients were allocated to groups with abnormal urinalysis (AU) or normal urinalysis (NU) based on urinalysis. It is also possible that the kidney impairment is due to drug nephrotoxicity. The medications of patients before admission included antibiotics (Moxifloxacin, Azithromycin, Amoxicillin) (30.1\%), antiviral drugs (Oseltamivir, Lopinavir, Arbidol) (33.8\%) and Chinese patent medicine (Lianhuaqingwen capsule, Isatis root granule) (10.8\%). However, there was no statistical difference in prehospital treatment between AU and NU group (Table 3). This data supported that the kidney impairment was caused by SARS-CoV-2 infection rather than treatment agents.

\section{Urinalysis abnormality correlates with severity of COVID-19}

Subsequently, in order to explore whether urinalysis correlates with disease severity, we compared the differences in other laboratory parameters between the AU and NU group. Firstly, we found that there 
was no difference in eGFR, Scr, BUN and serum uric acid between the two groups. Although the patients with AU had higher level of cystatin C, all the values were generally within normal range

(Figure 3A). Next, as shown in Figure 3B, the patients in AU group demonstrated remarkably higher level of liver injury related indicators, such as ALT, AST, GGT, $\alpha$-hydroxybutyrate dehydrogenase (HBDH), lactic dehydrogenase (LDH), alkaline phosphatase (ALP) and leucine aminopeptidase (LAP). And both serum prealbumin level and albumin-globulin ratio $(\mathrm{A} / \mathrm{G})$, markers related to liver synthetic capability and inflammatory state, were lower in AU group than in NU group, indicating possible liver inflammation. These data revealed that the patients with abnormal urinalysis might be subjected to liver damage. In addition, the patients in AU group usually presented with higher levels of inflammation related markers, including C-reactive protein (CRP), erythrocyte sedimentation rate (ESR), IL-6, serum ferritin (SF) and serum amyloid A (SAA) (Figure 3C). However, no significant differences were observed in major lymphocytes subpopulations, such as $\mathrm{CD}^{+} \mathrm{T}$ cells, $\mathrm{CD} 8^{+} \mathrm{T}$ cells, CD4/CD8, B cells and NK cells (Figure 3D). What's more, absolute count of peripheral lymphocyte of AU patients was significantly lower than that of NU patients, white blood cells (WBC) and neutrophils count were usually higher in patients in AU group (Figure 3E). There was no significant difference between the two groups in platelet, red blood cells (RBC) and hemoglobin (Figure 3E). As for coagulation profile, the AU patients had statistically higher levels of serum fibrinogen (FIB) and D-dimer than NU patients, indicating the worse coagulation function (Figure 3F). Collectively, these results indicated that the urinalysis abnormality correlates with severity of COVID-19, and urine routine test may be a good method for predicting the disease progression in COVID-19 patients.

\section{Discussion}


Here, we described the kidney impairment related manifestations among COVID-19 patients. Our results suggest kidney impairment are common in patients with SARS-CoV-2 infection, however, acute kidney injury occurs infrequently and primary symptoms were abnormal urinalysis, including proteinuria, hematuria, and leucocyturia. At the same time, urine routine test can be used as a good method that may be neglected to predict disease severity.

Several papers have demonstrated the renal impairment in COVID-19 patients. However, the proportion of increased Scr or incidence of AKI after SARS-CoV-2 infection was quite different among these studies. Some researches supported that SARS-CoV-2 infection cause significant renal impairment, however, some held an opposite view. We carefully read the relevant articles and found that the paradox may result from: (1) the difference in severity of illness; (2) the different scale of patient sampling. Further investigations are urgently needed to provide additional evidence of kidney impairment in COVID-19 patients.

In the current study, we enrolled 178 patients with confirmed SARS-CoV-2 infection including 52 cases admitted to ICU and 126 cases not. We found no patient exhibited a rise in Scr or Cystatin C and occurrence of AKI both in ICU group and non-ICU group. On the contrary, 54.2\% (45/83) patients who performed urine routine tests $(48.9 \%$ [87/178]) presented abnormal urinalysis, which was featured by proteinuria, hematuria and leucocyturia. These results reveal that renal impairment are common both in severe and non-severe COVID-19 patients, which is manifested by abnormal urine routine test rather than elevated level of Scr and AKI. Meanwhile, our study indicates that urinalysis is a better tool to screen kidney impairment. 
These two seemingly opposite results based on blood chemistry analysis and urinalysis respectively arouse our interest in exploring the underlying reasons. Firstly, although it's believed that SARS-CoV-2 infection causes kidney impairment, clinical symptoms were not obvious due to the powerful compensatory function of kidney. It's well known that serum levels of Scr and BUN will exceed the normal range only when more than $50 \%$ of kidney function has been lost. The extent of SARS-CoV-2 infection caused kidney impairment may be not enough to result in elevated plasma markers related renal function. In other words, "kidney impairment" doesn't lead to "kidney dysfunction" to a certain extent. Secondly, the primary damage is restricted in renal tubules rather than glomerulus (Figure 4). Several investigations revealed that SARS-CoV-2 gain entry into host cells by binding to ACE2 receptor on the host cell surface as the SARS-CoV does. Previous study of ACE2 immunostaining revealed that the mesangium and glomerular endothelium were negative for ACE2 and the distal tubules and collecting ducts showed weak cytoplasmic staining, whereas abundant staining was seen in the brush border of proximal tubular cells(22). Another two studies conducted by Xu et al and Qi's group pointed that proximal straight tubule cells were potential host cells targeted by SARS-CoV-2 by scRNA-seq analysis(12, 23). Indeed, such speculation has already been verified by Diao's group who reported immunohistochemistry of SARS-CoV-2 NP antigen in kidney specimens of six perished cases and found that NP expression was restricted in kidney tubules and absent in the glomerulus. Histopathological examination by H\&E staining in kidney specimens has proved acute tubular necrosis, luminal brush border sloughing and vacuole degeneration(24). Furthermore, another study on organ distribution of SARS-CoV in patients who died of SARS reported that SARS-CoV was detected in epithelial cells of distal convoluted renal tubules(25). Meanwhile, the pathological findings 
of kidney form autopsy in seven SARS subjects pinpointed that glomerular pathology was not observed in the kidneys, but acute tubular necrosis of varying degrees was found in all seven renal specimens(26).

So far, many parameters and models were established to predict the change of condition among COVID-19 patients. In order to evaluate whether urinalysis is useful to predict the disease severity, we then divided the patients into two groups based on their results of urine routine test on admission, named abnormal urinalysis group (AU) and normal urinalysis group (NU). Compared to control patients, patients in AU group presented no difference in BUN, Scr and eGFR, but had elevated cystatin C. In addition, we found that patients with abnormal urinalysis usually had more severe laboratory parameters, such as higher liver and heart injury index, including ALT, AST, ALP, LDH, HBDH, GGT and LAP, inflammation related markers, including CRP, IL-6, ESR, SAA and serum ferritin, blood routine indicator including WBC, neutrophils and lymphocytes, as well as coagulation related indicator including FIB and D-dimer. This analysis revealed that the patients with kidney impairment were generally in worse condition, including multiple organs or tissues injuries (liver, heart), inflammation and hypercoagulability state, which is probably because of low level of immunity and high viral load. In addition, our study indicates that urinalysis, a cheap, fast and easy way to perform, is an excellent method to predict the severe condition of COVID-19 patients.

Admittedly, there are several issues need to be addressed in this study. Firstly, we have no pathological evidence to confirm our speculation due to lack of specimen from biopsy or autopsy, such as H\&E and immunostaining. Secondly, the dynamic analysis of urinalysis was not performed in COVID-19 
patients due to many patients only have one urine routine test on admission. Finally, a larger sample size should be enrolled in our study. We will attempt to address these questions in our future research.

In summary, we provided the evidence that kidney impairment is common among COVID-19 patients, and its clinical manifestation is abnormal urinalysis, indicating that urine routine test is a better indicator to unveil potential kidney impairment than blood chemistry test. Furthermore, our results suggested that urinalysis is a useful tool to predict disease severity. Thus, we call for front-line healthcare workers pay more attention on kidney impairment in patients with SARS-CoV-2 infection and routinely monitor urinalysis to judge potential kidney impairment and evaluate disease severity.

\section{Contributors}

DH and HF designed the study. HZ, ZZ prepared figures and tables, and wrote the paper. ML, YD, WG,

LL, ZK, TY, CT, YG, RQ collected the data and analyzed the data. JL revised the paper. HW, SL and

SL participated in the discussion of this study and give suggestions on formulation of the manuscript.

\section{Disclosures}

The authors have no conflicts of interest.

\section{Acknowledgments}

This study was funded by the grants from the project of Thousand Youth Talents for D.H.; and from the

China National Natural Science Foundation (Nos. 31770983 and 81974249 to D.H., No. 81601747 to

S.L.). The authors thank all members of Wuhan Union Hospital for helpful suggestions and discussions 
in our study.

\section{References}

1. F. Zhou, Yu T., Du R., Fan G., Liu Y., Liu Z., et al. Clinical course and risk factors for mortality of adult inpatients with COVID-19 in Wuhan, China: a retrospective cohort study. Lancet. 2020;395(10229):1054-62.

2. Huang C, Y Wang, X Li, L Ren, J Zhao, Y Hu, et al. Clinical features of patients infected with 2019 novel coronavirus in Wuhan, China. Lancet (London, England). 2020;395(10223):497-506.

3. Chen N, M Zhou, X Dong, J Qu, F Gong, Y Han, et al. Epidemiological and clinical characteristics of 99 cases of 2019 novel coronavirus pneumonia in Wuhan, China: a descriptive study. Lancet (London, England). 2020;395(10223):507-13.

4. X. Yang, Yu Y., Xu J., Shu H., Xia J., Liu H., et al. Clinical course and outcomes of critically ill patients with SARS-CoV-2 pneumonia in Wuhan, China: a single-centered, retrospective, observational study. Lancet Respir Med. 2020;published online Feb 24, 2020. DOI:10.1016/S2213-2600(20)30079-5. 5. Wang D, B Hu, C Hu, F Zhu, X Liu, J Zhang, et al. Clinical Characteristics of 138 Hospitalized Patients With 2019 Novel Coronavirus-Infected Pneumonia in Wuhan, China. JAMA. 2020;published online Feb 7, 2020. DOI:10.1001/jama.2020.1585.

6. Al-Tawfiq JA. Asymptomatic coronavirus infection: MERS-CoV and SARS-CoV-2 (COVID-19). Travel medicine and infectious disease. 2020;published online Feb 27, 2020.

DOI:10.1016/j.tmaid.2020.101608:101608.

7. Chen D, W Xu, Z Lei, Z Huang, J Liu, Z Gao, et al. Recurrence of positive SARS-CoV-2 RNA in COVID-19: A case report. International journal of infectious diseases : IJID : official publication of the International Society for Infectious Diseases. 2020;published online Mar 5, 2020.

DOI:10.1016/j.ijid.2020.03.003.

8. Wu A, Y Peng, B Huang, X Ding, X Wang, P Niu, et al. Genome Composition and Divergence of the Novel Coronavirus (2019-nCoV) Originating in China. Cell host \& microbe. 2020;27(3):325-8.

9. Lu R, X Zhao, J Li, P Niu, B Yang, H Wu, et al. Genomic characterisation and epidemiology of 2019 novel coronavirus: implications for virus origins and receptor binding. Lancet (London, England). 2020;395(10224):565-74.

10. Z. Shen, Xiao Y., Kang L., Ma W., Shi L., Zhang L., et al. Genomic diversity of SARS-CoV-2 in Coronavirus Disease 2019 patients. Clin Infect Dis. 2020;published online Mar 4, 2020. DOI:10.1093/cid/ciaa203.

11. H. Zhang, Penninger J. M., Li Y., Zhong N., Slutsky A. S. Angiotensin-converting enzyme 2 (ACE2) as a SARS-CoV-2 receptor: molecular mechanisms and potential therapeutic target. Intensive Care Med. 2020;46(4):586-90.

12. F. Qi, Qian S., Zhang S., Zhang Z. Single cell RNA sequencing of 13 human tissues identify cell types and receptors of human coronaviruses. Biochem Biophys Res Commun. 2020;published online Mar 18,2020. DOI:10.1016/j.bbrc.2020.03.044.

13. Hoffmann M, H Kleine-Weber, S Schroeder, N Krüger, T Herrler, S Erichsen, et al. SARS-CoV-2 Cell Entry Depends on ACE2 and TMPRSS2 and Is Blocked by a Clinically Proven Protease Inhibitor. Cell. 2020; published online Mar 4, 2020. DOI:10.1016/j.cell.2020.02.052. 
14. Xiaoqiang Chai, Hu Longfei, Zhang Yan, Han Weiyu, Lu Zhou, Ke Aiwu, et al. Specific ACE2 Expression in Cholangiocytes May Cause Liver Damage After 2019-nCoV Infection. bioRxiv. 2020;doi:10.1101/2020.02.03.931766.

15. Cui Y, M Tian, D Huang, X Wang, Y Huang, L Fan, et al. A 55-Day-Old Female Infant infected with COVID 19: presenting with pneumonia, liver injury, and heart damage. The Journal of infectious diseases. 2020;published online Mar 17, 2020. DOl:10.1093/infdis/jiaa113.

16. Mo P, Y Xing, Y Xiao, L Deng, Q Zhao, H Wang, et al. Clinical characteristics of refractory COVID-19 pneumonia in Wuhan, China. Clinical infectious diseases : an official publication of the Infectious Diseases Society of America. 2020;published online Mar 16, 2020. DOl:10.1093/cid/ciaa270.

17. Guan WJ, ZY Ni, Y Hu, WH Liang, CQ Ou, JX He, et al. Clinical Characteristics of Coronavirus Disease 2019 in China. The New England journal of medicine. 2020;published online Feb 28, 2020. DOI:10.1056/NEJMoa2002032.

18. Zhen Li, Wu Ming, Guo Jie, Yao Jiwei, Liao Xiang, Song Siji, et al. Caution on Kidney Dysfunctions of 2019-nCoV Patients. medRxiv. 2020;doi:10.1101/2020.02.08.20021212.

19. Yichun Cheng, Luo Ran, Wang Kun, Zhang Meng, Wang Zhixiang, Dong Lei, et al. Kidney disease is associated with in-hospital death of patients with COVID-19. Kidney International.published online Mar 19, 2020. DOl:org/10.1016/j.kint.2020.03.005.

20. Lunwen Wang, Li Xun, Chen Hui, Yan Shaonan, Li Yan, Li Dong, et al. SARS-CoV-2 infection does not significantly cause acute renal injury: an analysis of 116 hospitalized patients with COVID-19 in a single hospital, Wuhan, China. medRxiv. 2020;doi:10.1101/2020.02.19.20025288.

21. Levey AS, LA Stevens, CH Schmid, YL Zhang, AF Castro, HI Feldman, et al. A new equation to estimate glomerular filtration rate. Annals of internal medicine. 2009;150(9):604-12.

22. Hamming I, W Timens, ML Bulthuis, AT Lely, G Navis, H van Goor. Tissue distribution of ACE2 protein, the functional receptor for SARS coronavirus. A first step in understanding SARS pathogenesis. The Journal of pathology. 2004;203(2):631-7.

23. Zou X, K Chen, J Zou, P Han, J Hao, Z Han. Single-cell RNA-seq data analysis on the receptor ACE2 expression reveals the potential risk of different human organs vulnerable to 2019-nCoV infection. Frontiers of medicine. 2020;published online Mar 12, 2020. DOI: 10.1007/s11684-020-0754-0.

24. Bo Diao, Wang Chenhui, Wang Rongshuai, Feng Zeqing, Tan Yingjun, Wang Huiming, et al. Human Kidney is a Target for Novel Severe Acute Respiratory Syndrome Coronavirus 2 (SARS-CoV-2) Infection. medRxiv. 2020;doi:10.1101/2020.03.04.20031120.

25. Ding $Y, L$ He, $Q$ Zhang, $Z$ Huang, $X$ Che, J Hou, et al. Organ distribution of severe acute respiratory syndrome (SARS) associated coronavirus (SARS-CoV) in SARS patients: implications for pathogenesis and virus transmission pathways. The Journal of pathology. 2004;203(2):622-30.

26. Chu KH, WK Tsang, CS Tang, MF Lam, FM Lai, KF To, et al. Acute renal impairment in coronavirus-associated severe acute respiratory syndrome. Kidney international. 2005;67(2):698-705.

\section{Figure legends}

\section{Figure 1. The flow chart of COVID-19 patients inclusion procedure.}

Figure 2. Pie chart illustrating COVID-19 patients exhibited abnormal urinalysis, including

proteinuria and hematuria. (A) Analysis of all the COVID-19 patients with urine routine test. (B) 
Analysis of proteinuria between ICU and non-ICU. (C) analysis of hematuria between ICU and non-ICU.

Figure 3. The comparison of laboratory parameters between abnormal urinalysis group and normal urinalysis group. Analysis of renal function (A), analysis of liver function (B), inflammation (C), lymphocytes subpopulations (D), blood routine (E) and coagulation function (F). Abbreviation: GGT, $\gamma$-glutamyl transpeptidase; HBDH: $\alpha$-hydroxybutyrate dehydrogenase.

Figure 4. Potential mechanisms of kidney impairment in COVID-19patients. SARS-CoV-2 can directly invade renal tubular epithelial cells via ACE2, a virus specific receptor that mainly expressed in proximal tubular cells, which potentially leading to host cells damage and death. At the same time, nephrotoxic drugs, hypoxia and infectious caused extensive immune activation, featured by cytokine storm and immune complex formation, were also factors related to tubular cells impairment. The impairment caused compromise of tubular integrity may be major reason that the occurrence of abnormal urinalysis. In addition, although no significant signs of kidney dysfunction and acute kidney injury, characterized by increased Scr, BUN and cystatin C, many known factors may provoke injury to the glomeruli, such as inflammation, nephrotoxic drugs and fluid overload. 
medRxiv preprint doi: https://doi.org/10.1101/2020.04.03.20051722; this version posted April 6, 2020. The copyright holder for this preprint (which was not certified by peer review) is the author/funder, who has granted medRxiv a license to display the preprint in perpetuity.

It is made available under a CC-BY-NC-ND 4.0 International license .

Table 1. Basic information and blood indicators related to kidney injury

\begin{tabular}{|c|c|c|c|c|}
\hline & $\begin{array}{c}\text { Total } \\
(\mathrm{N}=\mathbf{1 7 8})\end{array}$ & $\begin{array}{c}\mathrm{ICU} \\
(\mathrm{n}=52)\end{array}$ & $\begin{array}{r}\text { Non-ICU } \\
(n=126)\end{array}$ & ${ }^{\text {a } P}$ Value \\
\hline Age (Years) & $47.0(35.0-61.0)$ & $56.50(40.5-65.5)$ & $45.0(32.8-57.0)$ & $<0.001$ \\
\hline \multicolumn{5}{|l|}{ Sex } \\
\hline male & $72(40.4)$ & $32(61.5)$ & $40(31.7)$ & $<0.001$ \\
\hline female & 106 (59.6) & $20(38.5)$ & $86(68.3)$ & $<0.001$ \\
\hline \multicolumn{5}{|l|}{ Basic disease } \\
\hline hypertension & $29(16.3)$ & $15(28.8)$ & $14(11.1)$ & 0.004 \\
\hline hyperlipidemia & $3(1.7)$ & $1(1.9)$ & $2(1.6)$ & $>0.999$ \\
\hline diabetes & $19(10.7)$ & $9(17.3)$ & $10(7.9)$ & 0.066 \\
\hline digestive system & $10(5.6)$ & $4(7.7)$ & $6(4.8)$ & 0.481 \\
\hline respiratory system & $10(5.6)$ & $4(7.7)$ & $6(4.8)$ & 0.481 \\
\hline cardiovascular & $10(5.6)$ & $7(13.5)$ & $3(2.4)$ & 0.007 \\
\hline cancer & $8(4.5)$ & $1(1.9)$ & $7(5.6)$ & 0.440 \\
\hline urinary system & $4(2.2)$ & $2(3.8)$ & $2(1.6)$ & $>0.999$ \\
\hline Others & $23(12.9)$ & $5(9.6)$ & $18(14.3)$ & 0.398 \\
\hline non & $50(30.3)$ & $14(30.8)$ & $36(30.2)$ & 0.936 \\
\hline $\begin{array}{l}\text { Body temperature (max.) } \\
\text { ( } \square)\end{array}$ & $38.5(37.8-39.0)$ & $39(38.1-39.1)$ & $38.3(37.7-39.0)$ & 0.005 \\
\hline$>38$ & $110(61.8)$ & $39(75)$ & $71(56.3)$ & 0.020 \\
\hline $\operatorname{Ser}(\mu \mathrm{mol} / \mathrm{L})$ & $65.2(56.8-74.8)$ & $71.0(55.8-89.4)$ & $65.3(56.5-74.3)$ & 0.067 \\
\hline increase & 0 & 0 & 0 & $>0.999$ \\
\hline BUN (mmol/L) & $3.81(2.83-4.54)$ & $4.14(3.33-4.82)$ & $3.72(2.76-4.57)$ & 0.078 \\
\hline increase & $5(2.8)$ & $3(5.8)$ & $2(1.6)$ & 0.150 \\
\hline eGFR (ml/min) & $214.6(308.05)$ & $93.7(78.1-108.0)$ & $102.5(90.7-113.1)$ & 0.014 \\
\hline decrease & $42(23.6)$ & $19(36.5)$ & $23(18.3)$ & 0.009 \\
\hline Cystatin C (mg/L) & $0.77(0.68-0.86)$ & $0.81(0.73-0.88)$ & $0.77(0.68-0.87)$ & 0.155 \\
\hline increase & $3(1.7)$ & $1(1.9)$ & $2(1.6)$ & $>0.999$ \\
\hline
\end{tabular}

Note: Data are shown as median (IQR) or $\mathrm{n}(\%)$ as appropriate. $\mathrm{ICU}=$ intensive care unit, $\mathrm{Scr}=\mathrm{serum}$ creatinine, $\mathrm{BUN}=\mathrm{blood}$ urea nitrogen, eGFR=estimated glomerular filtration rate, IQR=interquartile range. ${ }^{a} P$ values indicate differences between ICU and Non-ICU patients. $P<0.05$ was considered statistically significant. 
medRxiv preprint doi: https://doi.org/10.1101/2020.04.03.20051722; this version posted April 6, 2020. The copyright holder for this preprint (which was not certified by peer review) is the author/funder, who has granted medRxiv a license to display the preprint in perpetuity.

It is made available under a CC-BY-NC-ND 4.0 International license .

Table 2. Abnormal urinalysis results in COVID-19

\begin{tabular}{|c|c|c|c|c|}
\hline & $\begin{array}{c}\text { Total } \\
(\mathrm{N}=\mathbf{8 3})\end{array}$ & $\begin{array}{c}\text { ICU } \\
(n=19)\end{array}$ & Non-ICU $(n=64)$ & ${ }^{a} P$ Value \\
\hline Abnormal urine routine & $45(54.2)$ & $15(78.9)$ & $30(46.9)$ & 0.014 \\
\hline \multicolumn{5}{|l|}{ Proteinuria } \\
\hline positive & $29(34.9)$ & $11(57.9)$ & $18(28.1)$ & 0.017 \\
\hline \multicolumn{5}{|l|}{ Hematuria } \\
\hline positive & $24(28.9)$ & $10(52.6)$ & $14(21.9)$ & 0.009 \\
\hline \multicolumn{5}{|l|}{ Leucocyturia } \\
\hline positive & $14(16.9)$ & $2(10.5)$ & $12(18.8)$ & 0.506 \\
\hline \multicolumn{5}{|l|}{ Urine glucose } \\
\hline positive & $0(0)$ & $0(0)$ & $0(0)$ & $>0.999$ \\
\hline \multicolumn{5}{|l|}{ Urine urothelial cell } \\
\hline positive & $10(12)$ & $1(5.3)$ & $9(14.1)$ & 0.441 \\
\hline
\end{tabular}

Note: Data are shown as n (\%). COVID-19=novel coronavirus disease 2019 , ICU=intensive care unit. ${ }^{\text {a }} P$ values indicate differences between $\mathrm{AU}$ and NU patients. $P<0.05$ was considered statistically significant.

Table 3. Prehospital medications between AU and NU groups

\begin{tabular}{lcccc}
\hline Medications before admission & Total $(\mathbf{N}=\mathbf{8 3})$ & $\mathbf{A U}(\mathbf{n}=\mathbf{4 5})$ & $\mathbf{N U}(\mathbf{n}=\mathbf{3 8})$ & ${ }^{\text {a }} \boldsymbol{P}$ Value \\
\hline Antibiotic & $25(30.1)$ & $11(35.5)$ & $14(26.9)$ & 0.411 \\
\hline Oseltamivir / Lopinavir & $12(14.5)$ & $7(22.6)$ & $5(9.6)$ & 0.119 \\
\hline Arbidol & $16(19.3)$ & $9(29.0)$ & $7(13.5)$ & 0.082 \\
\hline Chinese Patent Medicine & $9(10.8)$ & $3(9.7)$ & $6(11.5)$ & $>0.999$ \\
\hline
\end{tabular}

Note: Data are shown as $\mathrm{n}(\%)$. AU=abnormal urinalysis group, $\mathrm{NU}=$ normal urinalysis group. ${ }^{\text {a }} P$ values indicate differences between $\mathrm{AU}$ and NU patients. Chinese Patent Medicine consists of Lianhuaqingwen capsule and Isatis root granule. $P<0.05$ was considered statistically significant. 


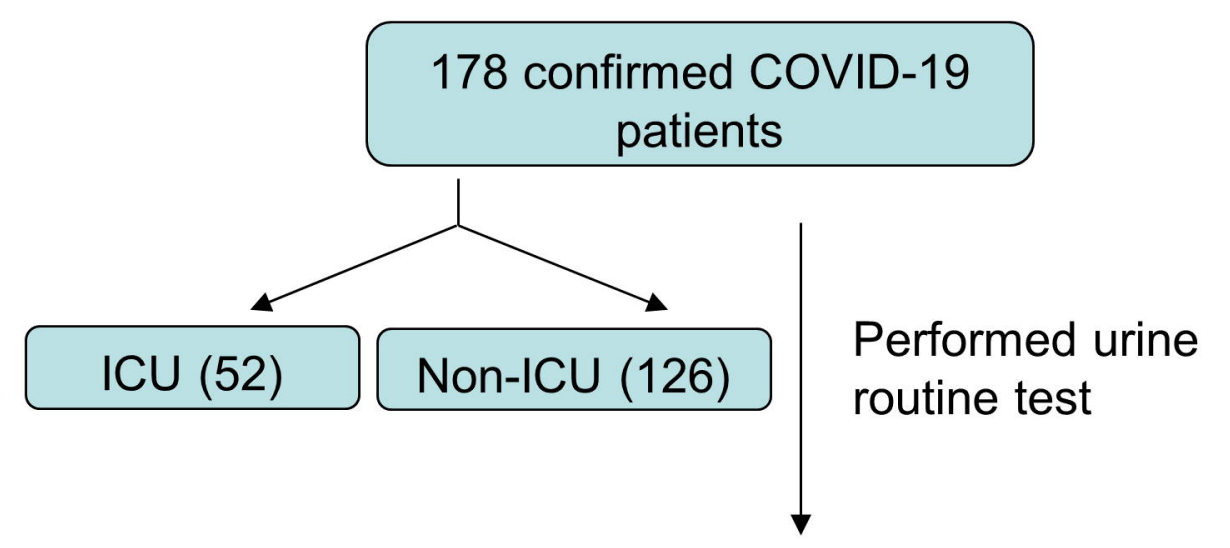

Urine routine test (87)

Excluded 4 patients

$\checkmark$ with renal diseases

Urine routine test (83)

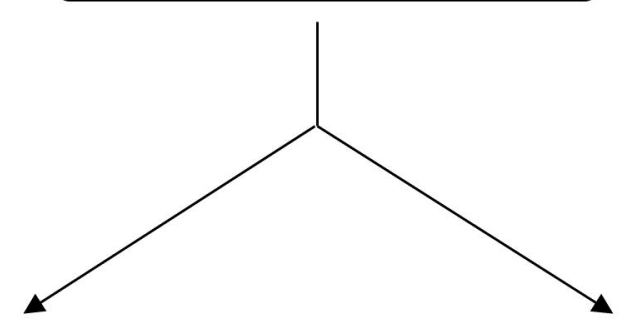

Abnormal urine routine (45) Normal urine routine (38) 
A Proteinuria

Hematuria

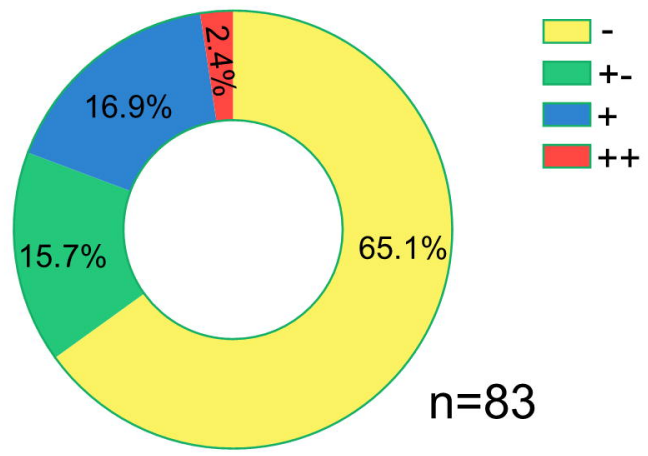

B
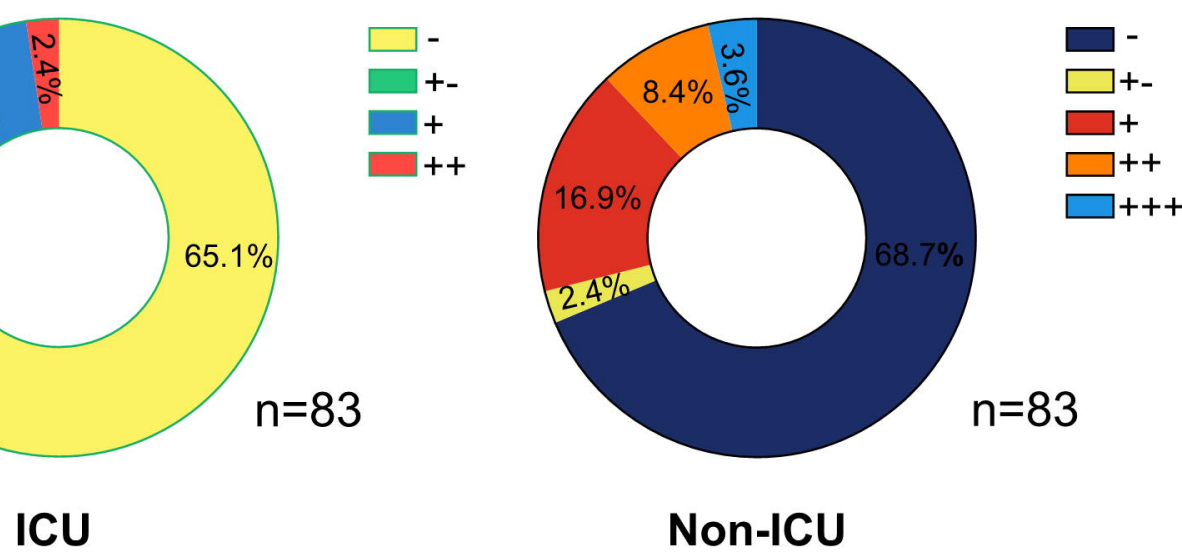

Non-ICU

\section{Proteinuria}

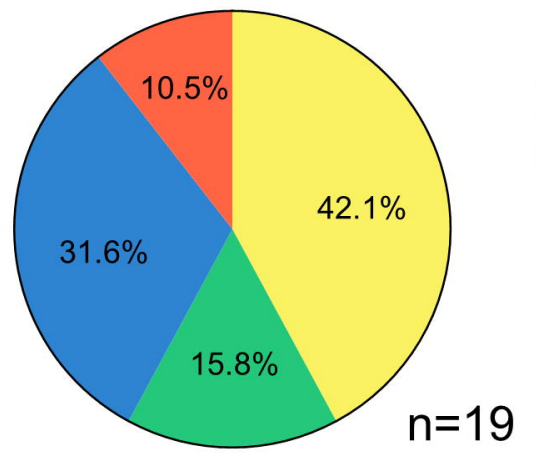

C

ICU

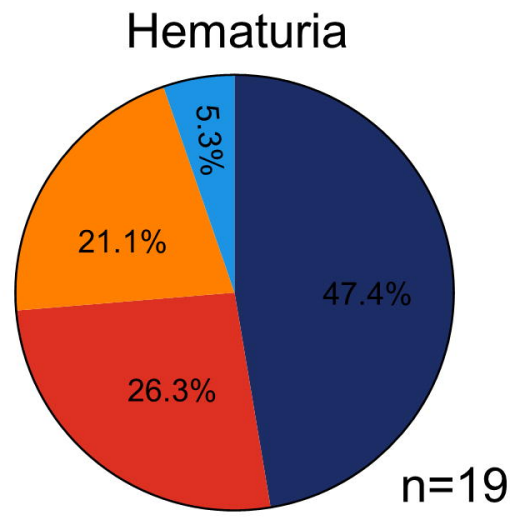

Proteinuria

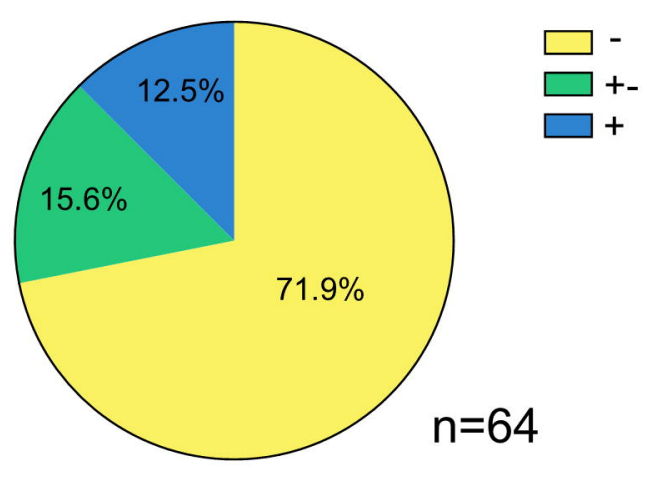

Non-ICU

Hematuria
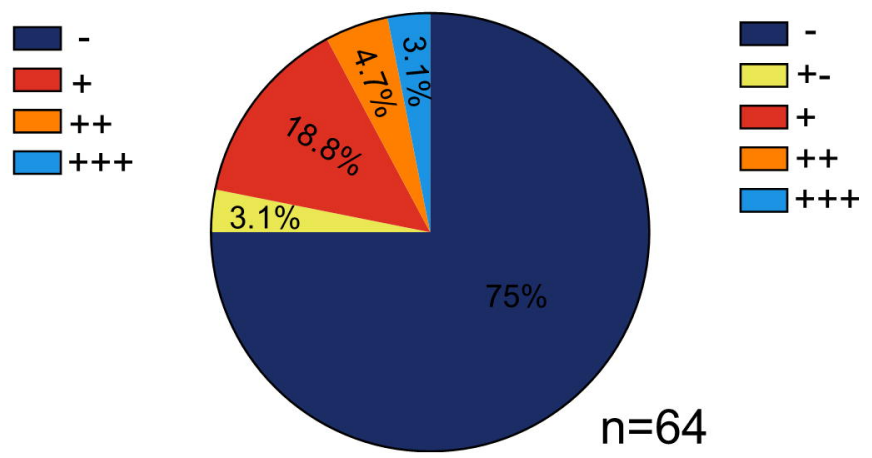
A

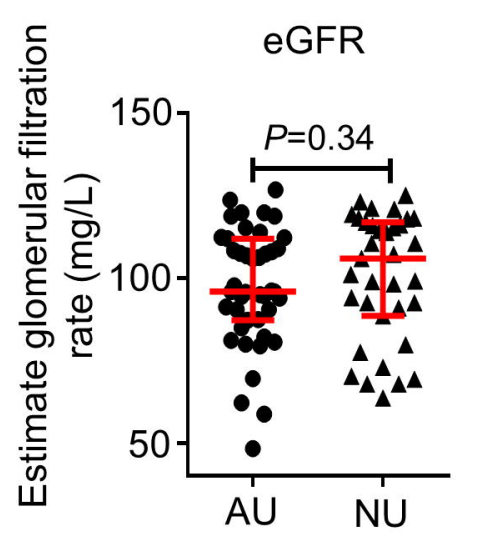

B

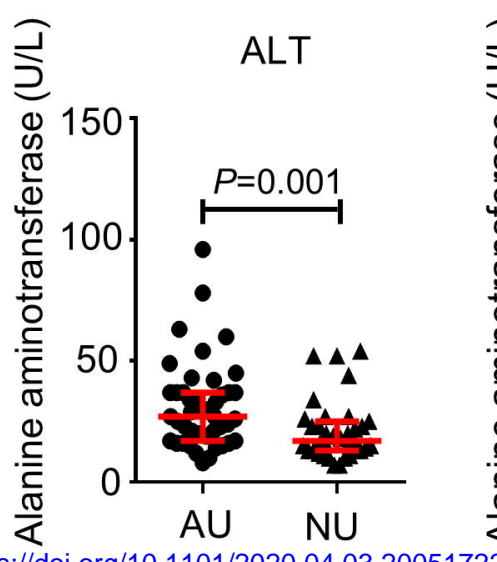

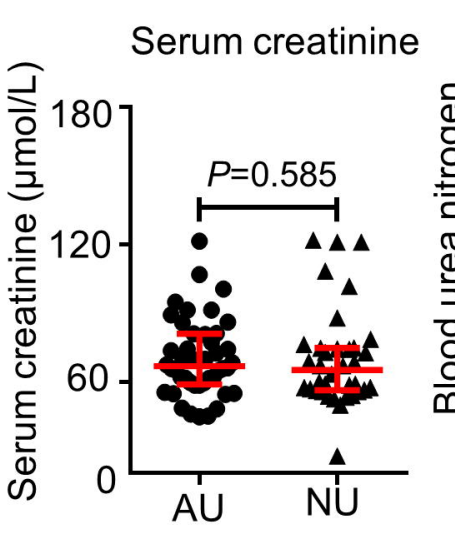

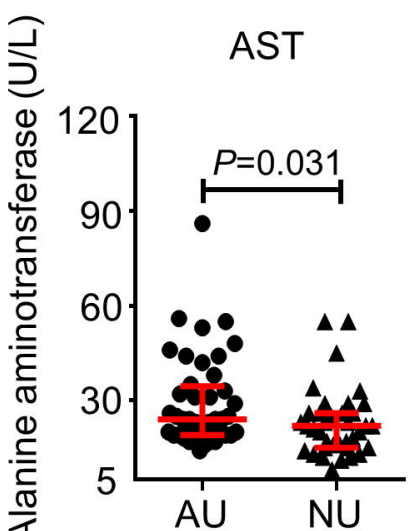

BUN
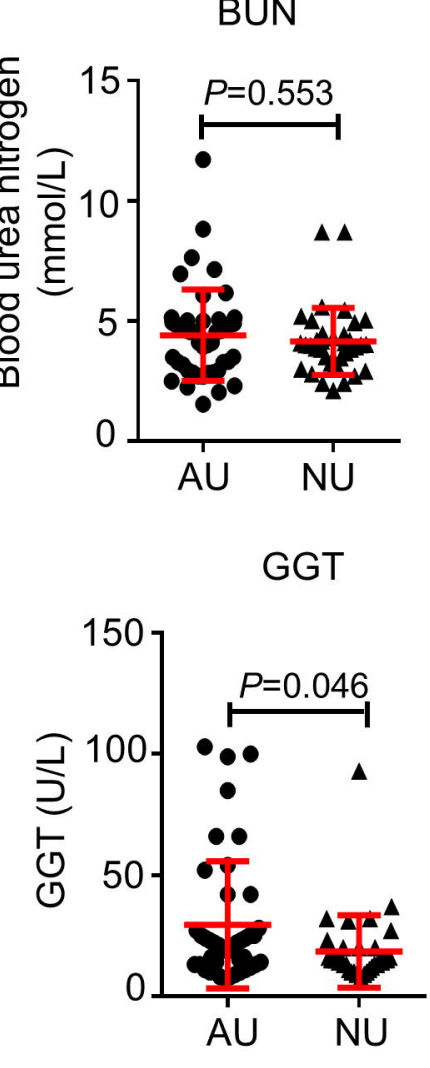

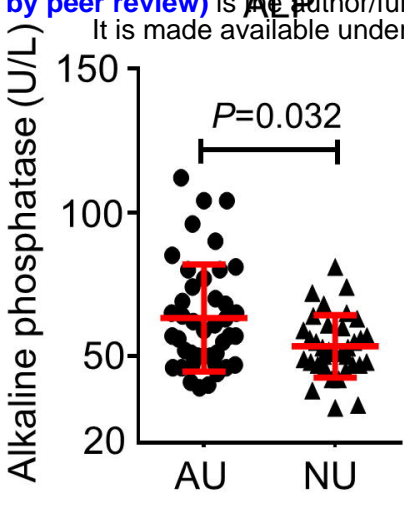

C

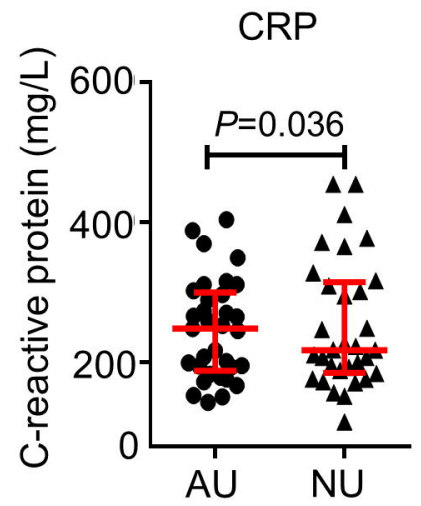

D

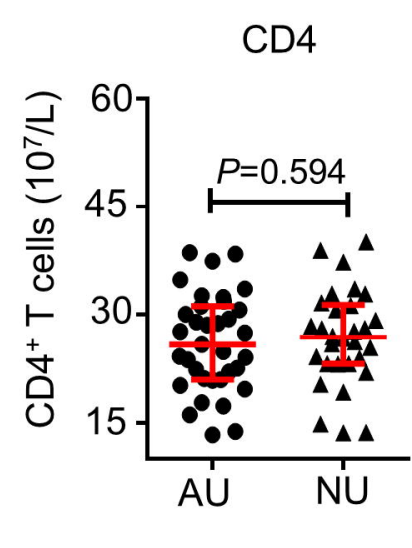

E

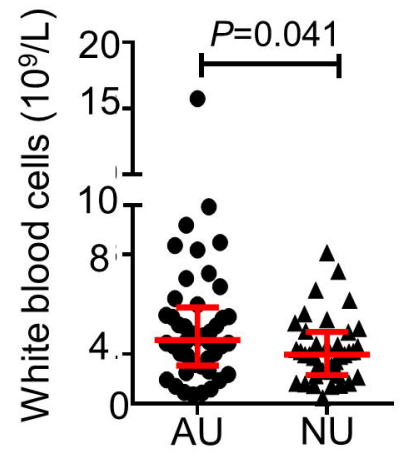

RBC

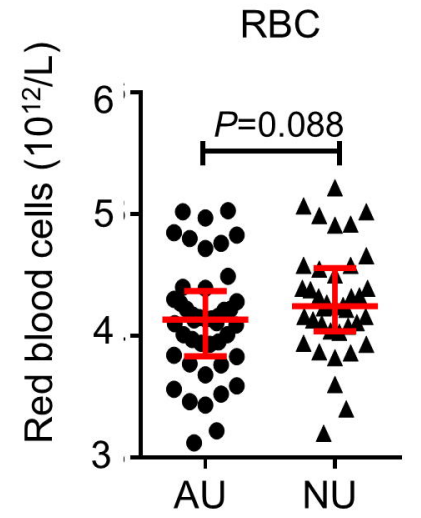

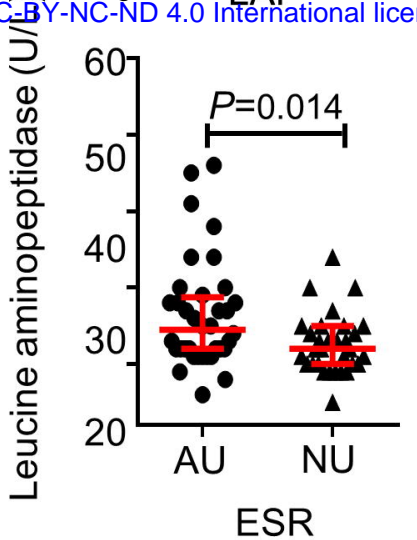
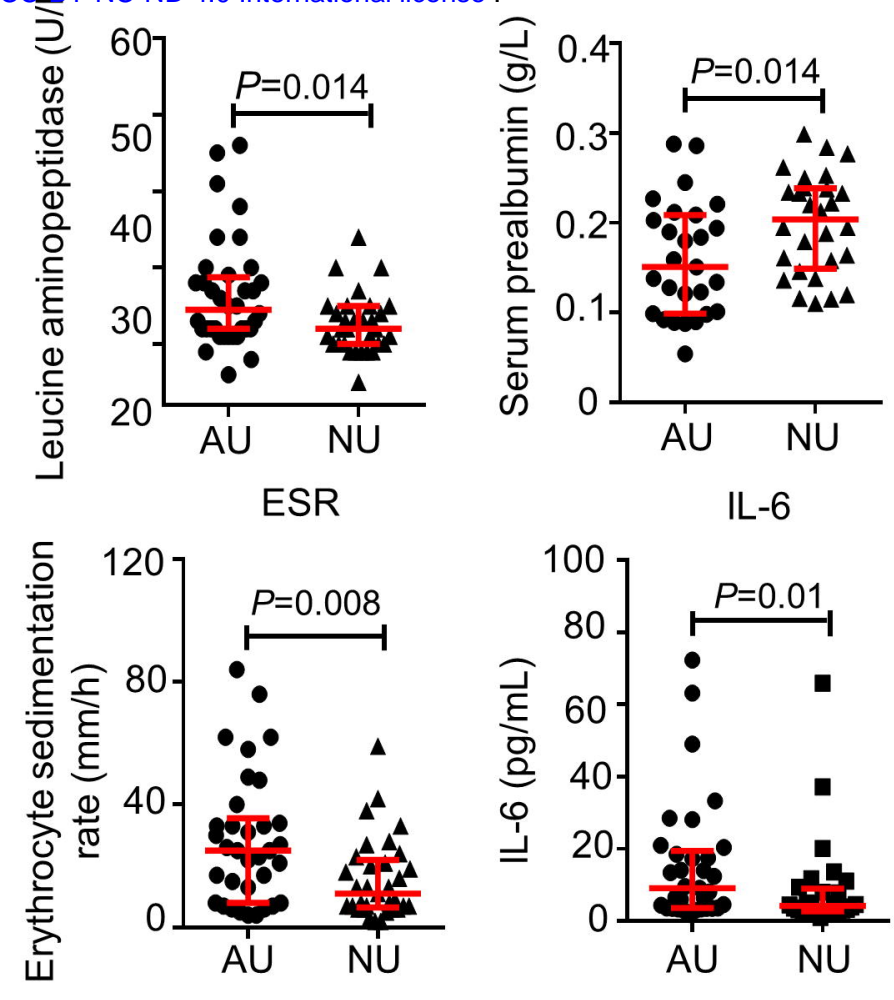

CD8

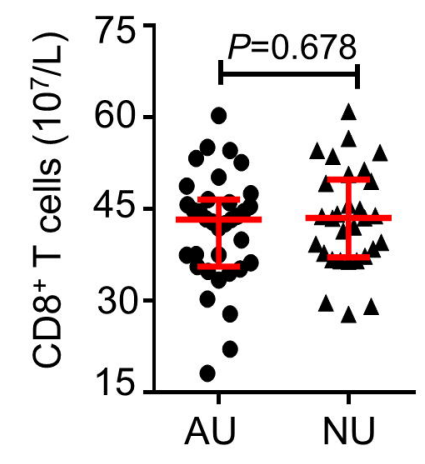

Neutrophils

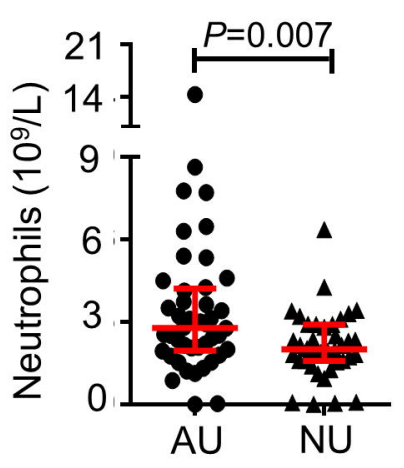

Hemoglobin

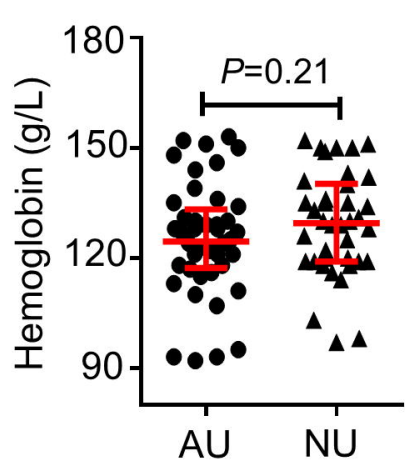

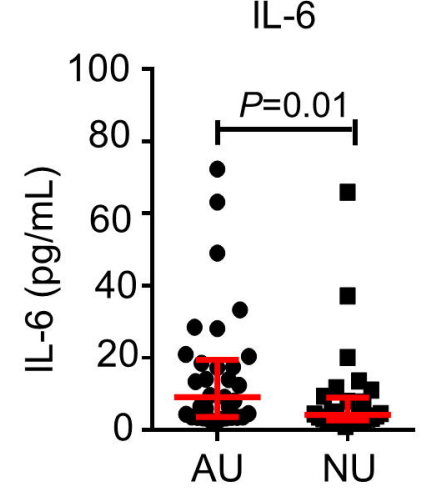

CD4/CD8

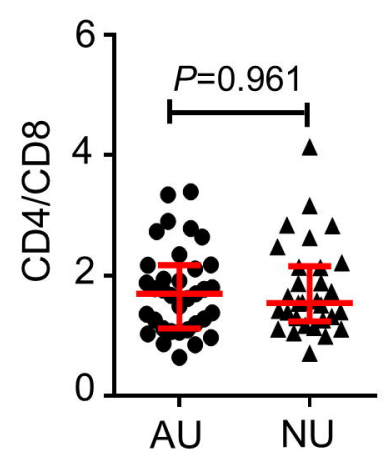

Lymphocytes

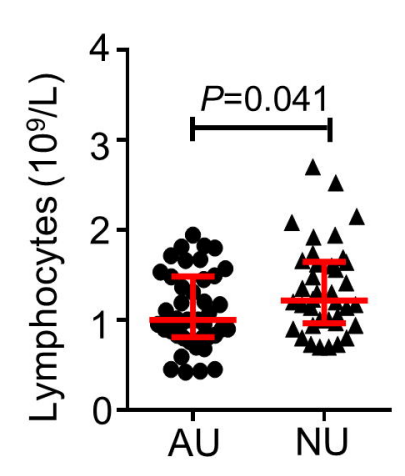

$\mathbf{F}$

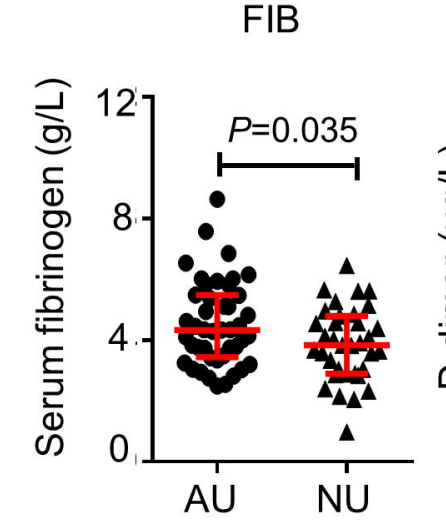

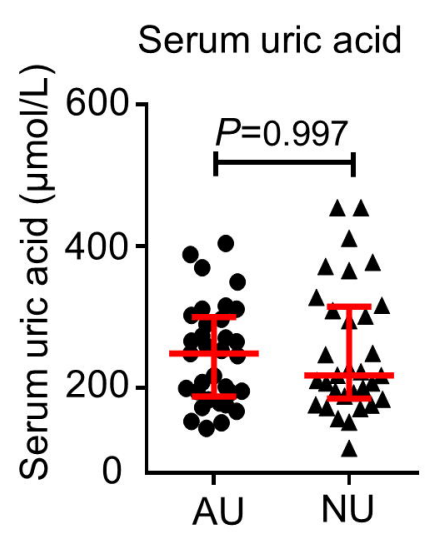

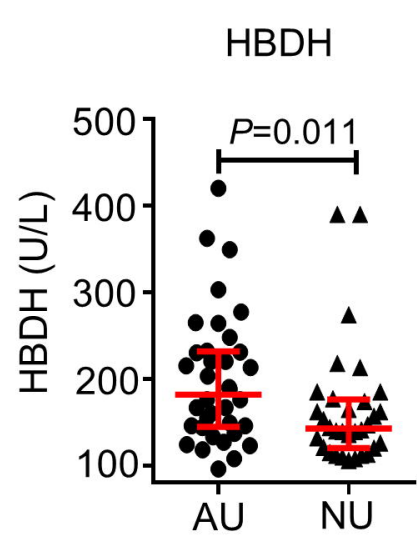

SA

Cystatin C
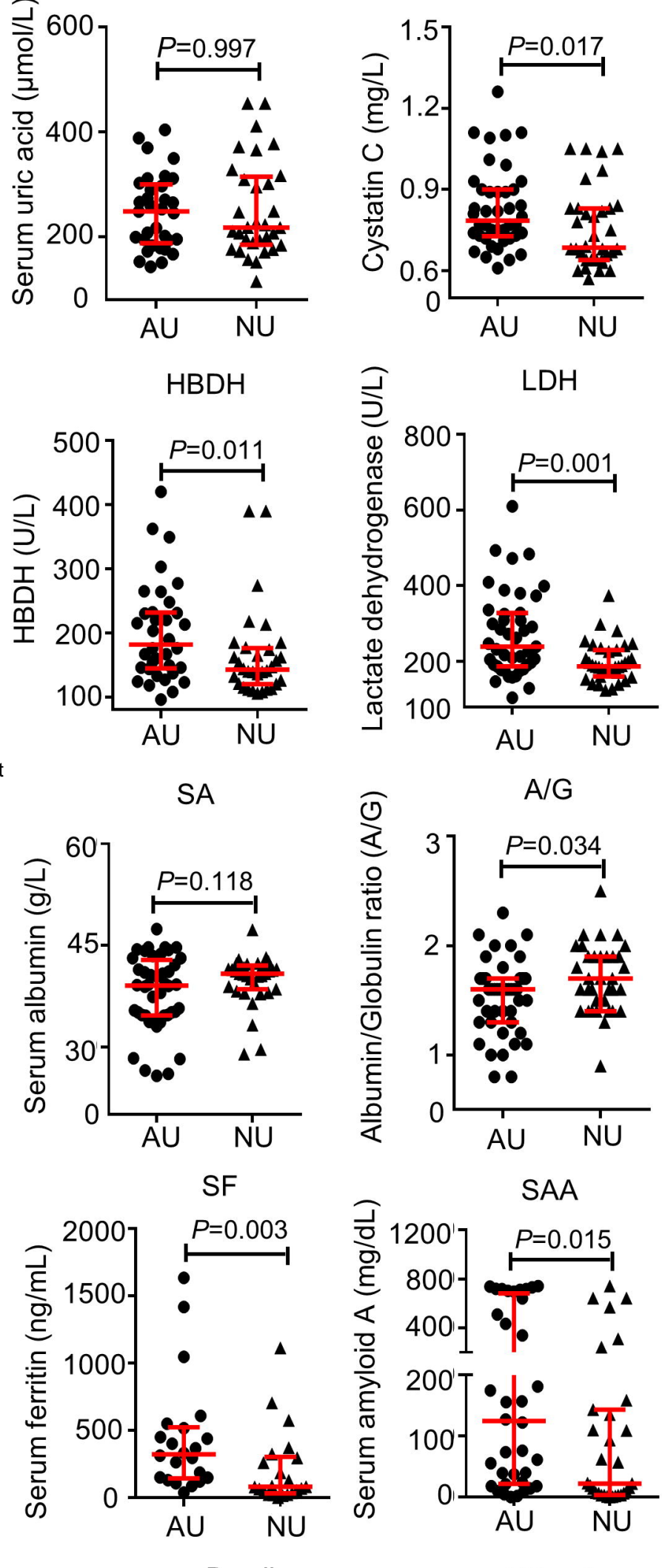

NK cell
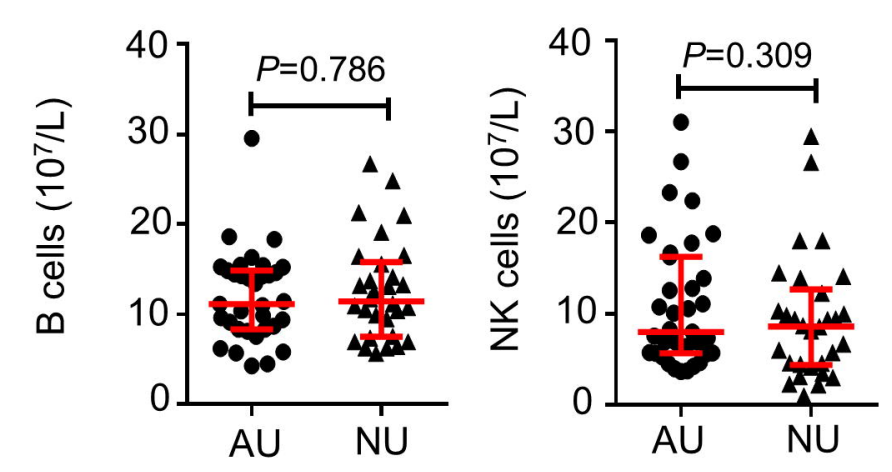

Eosinophil

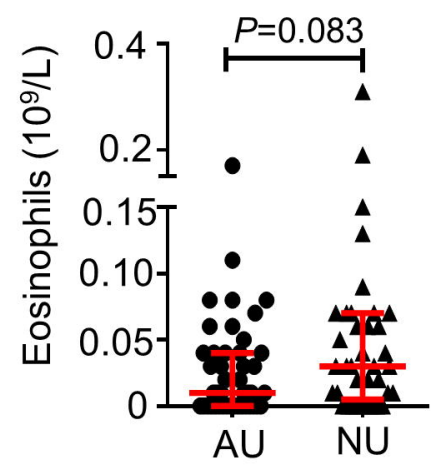

D-dimer
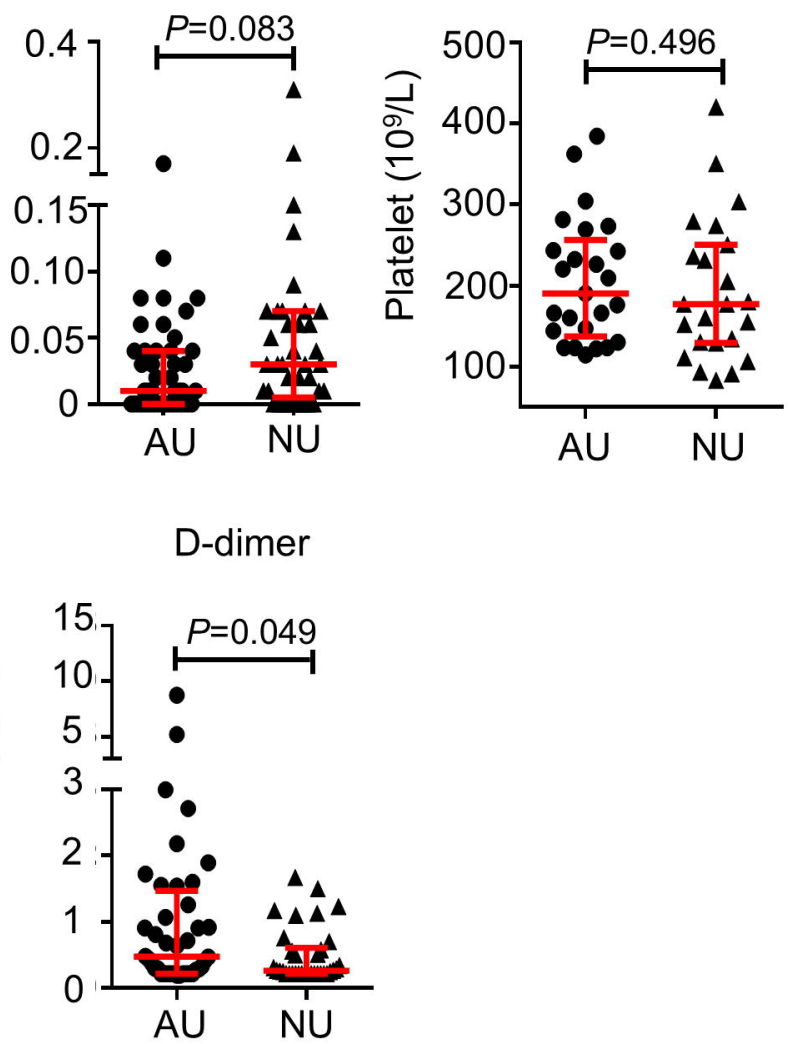


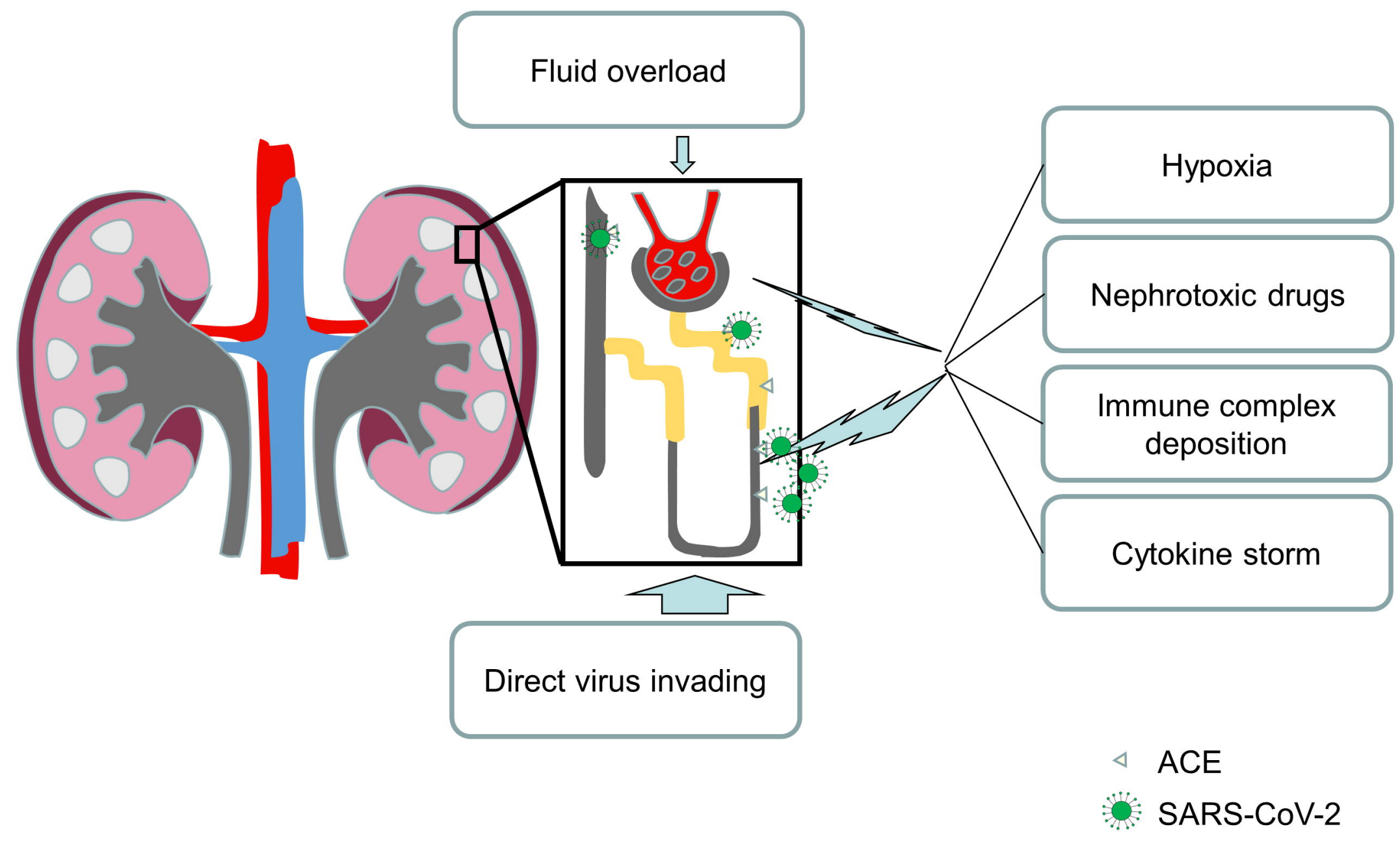

